\title{
Posicionamento palpebral superior e inferior em diferentes graus de rotação ocular ao longo do meridiano vertical
}

\author{
Upper and lower eyelid position in different degrees of downgaze and upgaze
}

\author{
Dante Auguusto Mastropietro ${ }^{1}$ \\ Luciano Ambrósio Alves ${ }^{2}$ \\ Antônio Augusto VelascoeCruz
}

Trabalho realizado na Divisão de Oftalmologia do Departamento de Oftalmologia, Otorrinolaringologia e Cirurgia de Cabeça e Pescoço da Faculdade de Medicina de Ribeirão Preto da Universidade de São Paulo - USP Ribeirão Preto (SP) - Brasil.

Residente da Divisão de Oftalmologia do Departamento de Oftalmologia, Otorrinolaringologia e Cirurgia de Cabeça e Pescoço da Faculdade de Medicina de Ribeirão Preto da USP - Ribeirão Preto (SP) - Brasil.

${ }^{2}$ Mestre em Ciências Médicas pela USP - Ribeirão Preto (SP) - Brasil.

${ }^{3}$ Professor titular da Divisão de Oftalmologia do Departamento de Oftalmologia, Otorrinolaringologia e Cirurgia de Cabeça e Pescoço da USP - Ribeirão Preto (SP) - Brasil.

Endereço para correspondência: Antonio Augusto Velasco e Cruz. Departamento de Oftalmologia, Otorrinolaringologia e Cirurgia de Cabeça e Pescoço. Av. Bandeirantes, 3.900 - Ribeirão Preto (SP) CEP 14049-900

E-mail: aavecruz@fmrp.usp.br.

Recebido para publicação em 03.08.2008

Última versão recebida em 12.10.2009

Aprovação em 04.11.2009

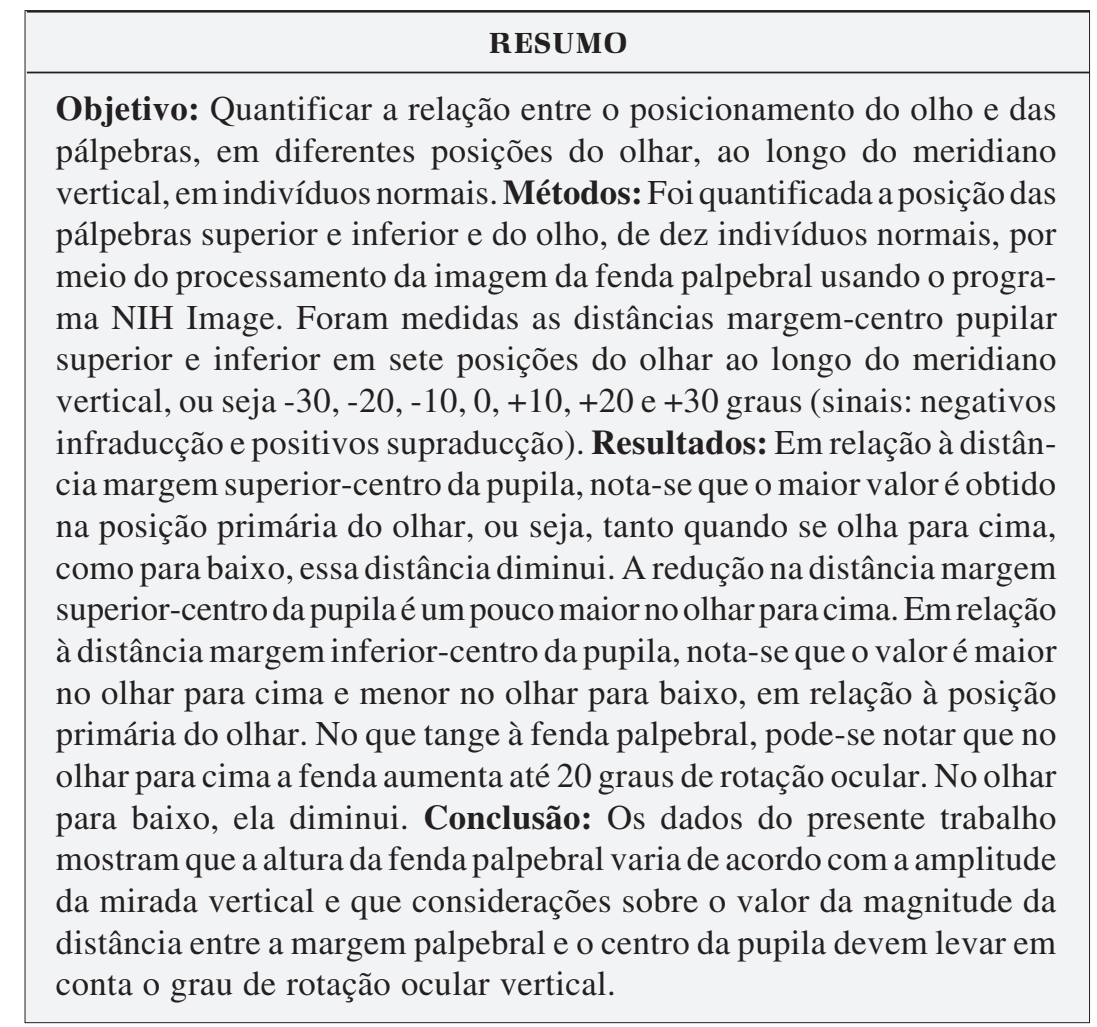

Descritores: Movimentos palpebrais; Pálpebras/fisiologia; Fixação, ocular

\section{INTRODUÇ̃̃OO}

A relação entre a posição do olho e as pálpebras é normalmente estudada na posição primária do olhar (PPO), por meio da quantificação das distâncias margem-centro pupilar superior (DMSP) e inferior (DMIP). Entretanto, há variações nessa relação, em diferentes posições do olhar ao longo do meridiano vertical, que são importantes e usualmente não quantificadas na prática clínica. Exemplos típicos são as variações na magnitude da ptose no olhar para baixo (aumento nas aponeuróticas e diminuição nas congênitas $)^{(1-4)}$ e a maior retração palpebral em infraversão que às vezes ocorre na orbitopatia de Graves (lid lag)(4-6).

O objetivo deste estudo é quantificar, em indivíduos normais, a relação entre o posicionamento do olho e das pálpebras em diferentes posições do olhar, ao longo do meridiano vertical, em pessoas normais. 


\section{MÉTODOS}

Foram estudadas 10 pessoas normais ( 5 do sexo masculino e 5 do feminino), com idades variando entre 17 e 42 anos (média de 33,1 \pm 9,02 anos). Foram excluídos sujeitos com história de doença sistêmica ou ocular, bem como usuários de lente de contato ou com história de cirurgia ocular prévia.

A posição palpebral superior e inferior foi quantificada processando-se a imagem da fenda palpebral nas diferentes posições da mirada vertical, usando o programa NIH Image (disponível em http://rsbweb.nih.gov/nih-image/). Para a obtenção das imagens da fenda palpebral, foi construído um sistema de captação composto por um arco metálico semicircular, orientado verticalmente e com a face côncava voltada para o sujeito a ter a fenda palpebral medida. Sobre esse arco, uma câmera de vídeo, conectada a um microcomputador por meio de uma placa capturadora (frame grabber), era movida de 10 em 10 graus superiormente (supraducção) e inferiormente (infraducção) à $\mathrm{PPO}$ até 30 graus de amplitude. As pessoas eram orientadas para olhar no centro da lente da câmera sem mover a cabeça, que estava fixada a uma mentoneira. Desse modo, obteve-se para cada indivíduo da pesquisa, sete imagens da fenda palpebral. Além da PPO (valor zero), três de 10 em 10 graus em supraducção, denominadas de $+10,+20$ e +30 , e três em infraducção designadas como -10, -20 e -30.

Para cada uma das imagens, mediu-se a DMSP, a DMIP e a altura da fenda palpebral que é a soma algébrica da DMSP e DMIP. Análise de variância unifatorial (ANOVA) para medidas repetidas, com posterior análise pelo teste de Tukey, foram usadas para a comparação dos valores medidos em cada uma das posições do olhar.

\section{RESULTADOS}

A figura 1 mostra os valores médios das DMSP, DMIP e da fenda palpebral ao longo da mirada vertical. A ANOVA indicou que há diferenças significativas nas três variáveis em função da posição do olhar: DMSP, $\mathrm{F}=332,36$; $\mathrm{p}<0,00001$; DMIP, $\mathrm{F}=877,19 ; \mathrm{p}<0,00001$; fenda palpebral, $\mathrm{F}=1268,75, \mathrm{p}<0,00001$.

No que tange à DMSP, nota-se que o maior valor é obtido na PPO (4,1 mm), ou seja, tanto quando se olha para cima, como para baixo, essa distância diminui. A redução na DMSP é um pouco maior no olhar para cima. De fato, em supraducção, o teste de Tukey revelou que a DMSP em 20 graus $(3,13 \mathrm{~mm})$ e 30 graus (2,55 mm) são significativamente diferentes da medida da PPO. Já em infraducção a variação é menor e apenas em 30 graus a DMSP (3,1 mm) é significativamente diferente da em PPO.

Em relação à DMIP, o maior valor é obtido no olhar para cima (9,0 mm em +30 graus) e o menor no olhar para baixo (4,8 mm em -30 graus). O teste de Tukey mostrou que tanto no olhar para baixo como para cima já há diferenças significativas em relação à medida na PPO $(6,6 \mathrm{~mm})$ a partir de 20 graus $(-20=5,3 \mathrm{~mm} ;-30=4,8 \mathrm{~mm} ;+20=8,5 \mathrm{~mm},+30=9,0 \mathrm{~mm})$.
As variações da fenda palpebral refletem as modificações de ambas as pálpebras e são bem descritas por uma função do tipo sigmóide, isto é, que tende à saturação nos pontos extremos da curva. É fácil notar que a fenda aumenta no olhar para cima e diminui no olhar para baixo. O teste de Tukey revelou que o valor da PPO (10,8 mm) só foi significativamente diferente dos obtidos em infraducção em -20 (8,7 mm) e em -30 graus (7,8 mm).

A figura 2 indica as variações médias que ocorrem com a DMSP, DMIP e fenda palpebral, em relação à PPO. Pode-se notar que as variações que ocorrem na DMIP são maiores que as das DMSP.

\section{DISCUSSÃO}

Os estudos que têm sido feitos sobre a relação óculo-palpebral buscam, na grande maioria dos casos, o entendimento das características dinâmicas dos movimentos palpebrais de origem ocular. Já foi demonstrado que tanto a duração como a amplitude do movimento palpebral superior e do olho no olhar para baixo são bastante semelhantes ${ }^{(7)}$.Também o movimento da pálpebra na direção superior é mais lento e menor que o ocular provavelmente porque o movimento palpebral no olhar para baixo é devido unicamente ao relaxamento do músculo levantador da pálpebra superior e atuação de forças elásticas passivas palpebrais $^{(8)}$. Essa idéia foi reafirmada com estudos em eletromiografia e dinâmica palpebral ${ }^{(9)}$.

Esses estudos não abordaram o principal parâmetro clínico, que é a relação entre a margem palpebral e o centro pupilar. $\mathrm{O}$ único trabalho que trata dessa questão foi realizado utilizando uma régua milimetrada e medidas no plano frontal ${ }^{(10)}$. Apesar de o método ter sido bastante rudimentar e diferente do utilizado no presente estudo, os resultados foram semelhantes, ou seja, a DMSP diminui tanto no olhar para cima, quanto no olhar para baixo; a DMIP aumenta no olhar para cima e diminuiu no olhar para baixo; e a altura da fenda palpebral também aumenta no olhar para cima e diminui no olhar para baixo.

Os valores médios encontrados indicam que, com 30 graus de supraducção, é normal ocorrer uma diminuição de 1,57 \pm 0,29 mm na DMSP. Já na infraducção, a mesma magnitude de rotação ocular induz uma redução menor da DMSP, da ordem de 1,06 $\pm 0,19 \mathrm{~mm}$.

Esses valores têm importância para o diagnóstico do mal posicionamento palpebral superior que ocorre nas variações do olhar vertical. Assim, diminuições maiores que 1,06 \pm 0,19 mm da DMSP no olhar para baixo sugerem ptose. Esse tipo de ptose, que na literatura americana é denominado "downgaze ptosis", tipicamente ocorre nas desinserções da aponeurose do músculo levantador da pálpebra superior ${ }^{(3)}$ e é fonte de queixa específica durante a leitura. O contrário ocorre nas ptoses congênitas, nas quais o fenômeno é oposto. Nesses casos, no olhar para baixo, devido ao movimento palpebral restrito, a DMSP não diminui, podendo mesmo ser maior que a do olho não acometido. 

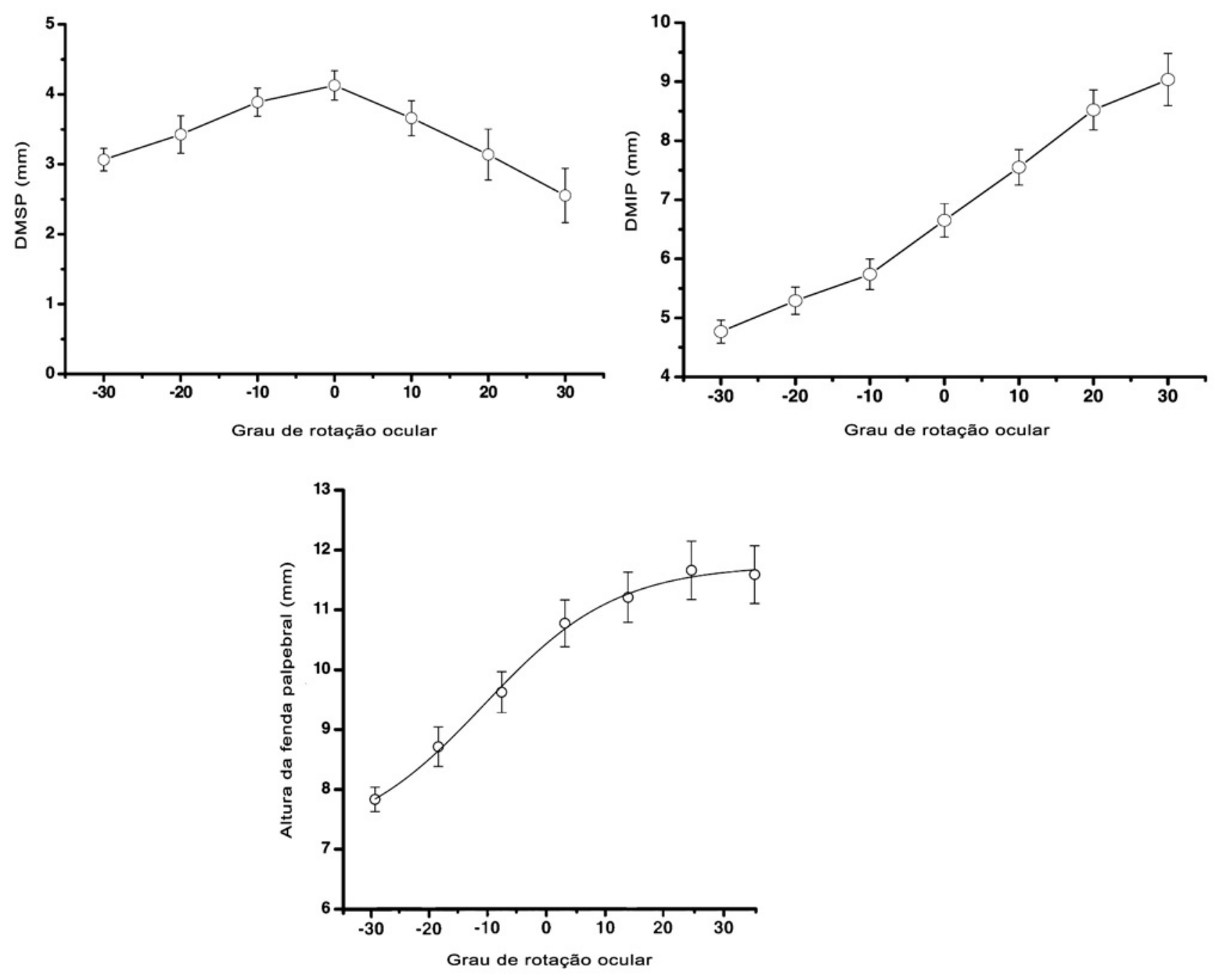

Figura 1 - Distâncias entre as margens palpebrais superior (esquerda) e inferior (direita) ao centro da pupila, bem como altura da fenda palpebral (abaixo) em diferentes graus de rotação ocular ao longo do meridiano vertical. Valores negativos de rotação equivalem à infraducção, valores positivos, supraducção. A posição primária do olhar é representada pelo valor 0 . Os símbolos representam valores médios \pm erro padrão. A curva no gráfico da altura da fenda palpebral corresponde a uma função sigmóide tipo $y=A 1+\left(A 2-A 1 / 1+10^{(L O G \times 0-x) p}\right)$.

Na retração palpebral secundária à orbitopatia de Graves, o comportamento da pálpebra superior algumas vezes é o mesmo do lagoftalmo cicatricial, ou seja, aumenta no olhar para baixo devido à restrição de movimento. Dito de outra maneira, a típica diminuição da DMSP em infraducção não ocorre. É o chamado "lid lag", ou sinal de von Graefe ${ }^{(6)}$. Nem sempre esse sinal está presente e frequentemente, apesar de a pálpebra superior estar retraída, a DMSP diminui ${ }^{(4,6)}$.

Todas estas observações poderão ser confirmadas em pesquisas futuras, usando a metodologia proposta no presente estudo.

Os valores médios também indicam que um aumento de 2,39 $\pm 0,36$ mm na DMIP a 30 graus de supraducção é normal, bem como uma redução de $1,88 \pm 0,33 \mathrm{~mm}$ na infraducção à mesma magnitude de rotação ocular.
A partir desses valores também é possível balizar o diagnóstico do mal posicionamento palpebral inferior que ocorre nas variações do olhar vertical. Se, no olhar para cima, a DMIP aumenta mais que $2,39 \pm 0,36 \mathrm{~mm}$, pode haver um indício de que a pálpebra inferior que se movimenta aquém do esperado. Isso acontece, por exemplo, nas fibroses cicatriciais pós-fratura orbitária, blefaroplastia ou exérese de tumores, entre outros ${ }^{(11-12)}$. Da mesma forma, se no olhar para baixo a DMIP cair além de 1,88 \pm $0,33 \mathrm{~mm}$, a pálpebra pode estar mais fixa que o normal, podendose encontrar ptose inversa da pálpebra inferior ${ }^{(13)}$.

Concluindo, os dados do presente trabalho fornecem elementos quantitativos preliminares para a avaliação da variação da amplitude de movimento palpebral na mirada vertical de indivíduos normais, um exame pouco valorizado, mas de clara importância semiótica. 

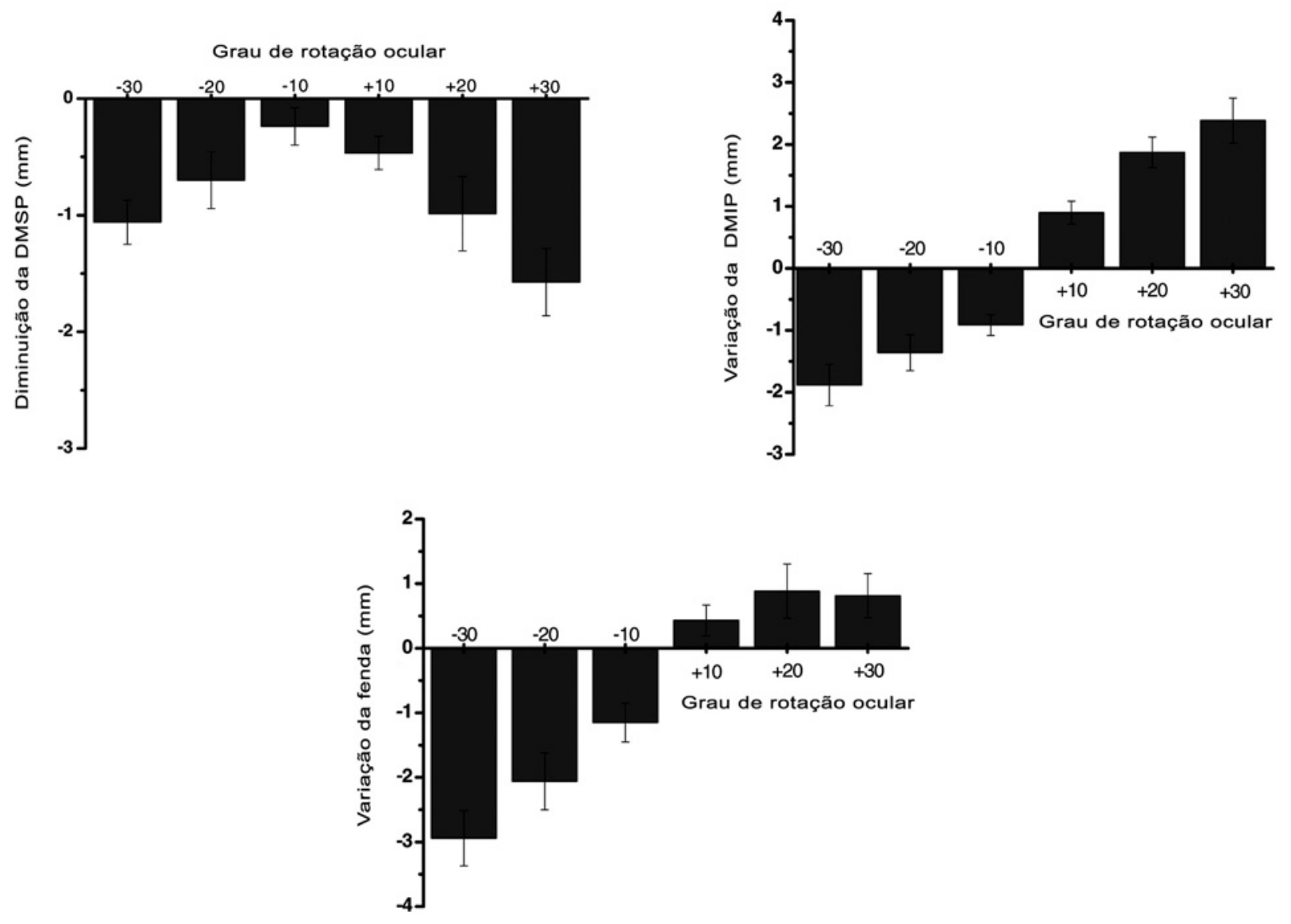

Figura 2 - Variação entre as margens palpebrais superior (esquerda) e inferior (direita) ao centro da pupila, bem como da altura da fenda palpebral (abaixo) em diferentes graus de rotação ocular ao longo do meridiano vertical, em relação à posição primária do olhar. Valores negativos de rotação equivalem à infraducção, valores positivos, supraducção. Os símbolos representam valores médios \pm erro padrão.

\section{ABSTRACT}

Purpose: To quantify the relation between eye and eyelids placement in different positions during downgaze and upgaze in healthy subjects. Methods: The position of the eye and eyelids of 10 healthy individuals was quantified by palpebral fissure image processing with NIH Image software. Upper and lower mid-pupil margin distance was measured in 7 positions: $-30,-20,-10,0,+10,+20$ and +30 degrees (positive signs correspond to upgaze and negative signs to downgaze) along vertical meridian. Results: The upper mid-pupil margin distance decreases in upgaze and even more in downgaze. The lower midpupil margin distance increases in upgaze and decreases in downgaze. About the palpebral fissure height, it gets larger in upgaze until 20 degrees and smaller in downgaze. Conclusion: Our data showed that the interpalpebral fissure height varies with vertical meridian gaze amplitude and that considerations about mid-pupil margin distance must consider the amount of upgaze or downgaze.
Keywords: Eyelids movements; Eyelids/physiology; Fixation, ocular

\section{REFERÊNCIAS}

1. Dryden RM, Kahanic DA. Worsening of blepharoptosis in downgaze. Ophthal Plast Reconstr Surg. 1992;8(2):126-9.

2. Patipa M. Visual field loss in primary gaze and reading gaze due to acquired blepharoptosis and visual field improvement following ptosis surgery. Arch Ophthalmol. 1992;110(1):63-7.

3. Wojno TH. Downgaze ptosis. Ophthal Plast Reconstr Surg. 1993;9(2):83-8; discussion 88-9.

4. Guimarães FC, Cruz AA. Palpebral fissure height and downgaze in patients with Graves upper eyelid retraction and congenital blepharoptosis. Ophthalmology. 1995;102(8):1218-22.

5. Feldon SE, Levin L. Graves' ophthalmopathy: V. Aetiology of upper eyelid retraction in Graves' ophthalmopathy. Br J Ophthalmol. 1990;74(8):484-5.

6. Gaddipati RV, Meyer DR. Eyelid retraction, lid lag, lagophthalmos, and von Graefe's sign quantifying the eyelid features of Graves' ophthalmopathy. Ophthalmology. 2008;115(6):1083-8.

7. Becker W, Fuchs AF. Lid-eye coordination during vertical gaze changes in man and monkey. J Neurophysiol. 1988;60(4):1227-52.

8. Fuchs AF, Becker W, Ling L, Langer TP, Kaneko CR. Discharge patterns of 
levator palpebrae superioris motoneurons during vertical lid and eye movements in the monkey. J Neurophysiol. 1992;68(1):233-43.

9. Evinger C, Manning KA, Sibony PA. Eyelid movements. Mechanisms and normal data. Invest Ophthalmol Vis Sci. 1991;32(2):387-400.

10. Stoller SH, Meyer DR. Quantitating the change in upper eyelid position during downgaze. Ophthalmology. 1994;101(9):1604-7.
11. Small RG, Scott M. The tight retracted lower eyelid. Arch Ophthalmol. 1990; 108(3):438-44.

12. Patipa M. The evaluation and management of lower eyelid retraction following cosmetic surgery. Plast Reconstr Surg. 2000;106(2):438-53; discussion 454-9.

13. Bartley GB, Frueh BR, Holds JB, Linberg JV, Patel BC, Hawes MJ. Lower eyelid reverse ptosis repair. Ophthal Plast Reconstr Surg. 2002;18(1):79-83. 